\title{
IMPROVED APPROXIMATIONS FOR THE DISTRIBUTIONS OF MULTINOMIAL GOODNESS-OF-FIT STATISTICS BASED ON $\phi$-DIVERGENCE UNDER NONLOCAL ALTERNATIVES
}

\author{
Pan Ei Htwe*, Nobuhiro Taneichi** and Yuri Sekiya***
}

\begin{abstract}
Zografos et al. (1990) introduced the $\phi$-divergence family of statistics $C_{\phi}$ to the goodness-of-fit test. The $\phi$-divergence family of statistics $C_{\phi}$ includes the power divergence family of statistics proposed by Cressie and Read (Cressie and Read (1984) and Read and Cressie (1988)) as a special case. Sekiya and Taneichi (2004) derived the multivariate Edgeworth expansion assuming a continuous distribution for the distributions of power divergence statistics under a nonlocal alternative hypothesis. In this paper, we consider an expansion for the family of general $\phi$-divergence statistics $C_{\phi}$. We derive the multivariate Edgeworth expansion assuming a continuous distribution for the distribution of $C_{\phi}$ under a nonlocal alternative hypothesis. By using the expansion, we propose a new approximation for the power of the statistic $C_{\phi}$. We numerically investigate the accuracy of the approximation when two types of concrete $\phi$-divergence statistics are applied. By the numerical investigation, we show that the present approximation is a good approximation especially when alternative hypotheses are distant from the null hypothesis.
\end{abstract}

Key words and phrases: Goodness-of-fit test, multinomial distribution, multivariate Edgeworth expansion, nonlocal alternative hypothesis, $\phi$-divergence statistic.

\section{Introduction}

Csiszár (1967) and Ali and Silvey (1966) proposed that the $\phi$-divergence measure between discrete distributions $\boldsymbol{p}=\left(p_{1}, \ldots, p_{k}\right)^{\prime}$ and $\boldsymbol{q}=\left(q_{1}, \ldots, q_{k}\right)^{\prime}$ is defined by

$$
D_{\phi}(\boldsymbol{p}, \boldsymbol{q})=\sum_{j=1}^{k} q_{j} \phi\left(\frac{p_{j}}{q_{j}}\right)
$$

where

$$
\begin{aligned}
& \phi(0)=\lim _{t \rightarrow+0} \phi(t), \\
& 0 \phi\left(\frac{x}{0}\right)=\lim _{y \rightarrow+0} y \phi\left(\frac{x}{y}\right)=x \lim _{t \rightarrow+0} t \phi\left(\frac{1}{t}\right), \quad \text { if } \quad x>0,
\end{aligned}
$$

Received July 3, 2010. Revised July 15, 2011. Accepted August 2, 2011.

*Department of System Information Science, Graduate School of Science and Engineering, Kagoshima University, 1-21-35 Korimoto, Kagoshima 890-0065, Japan. Email: paneihtwe06@gmail.com

**Department of Mathematics and Computer Science, Graduate School of Science and Engineering (Science course), Kagoshima University, 1-21-35 Korimoto, Kagoshima 890-0065, Japan. Email: taneichi@sci.kagoshima-u.ac.jp

***Kushiro Campus, Hokkaido University of Education, 1-15-55 Shiroyama, Kushiro 085-8580, Japan. Email: sekiya@kus.hokkyodai.ac.jp 
$0 \phi(0 / 0)=0$ and $\phi(t)$ being a real convex function for $t>0$.

Let $\boldsymbol{X}=\left(X_{1}, \ldots, X_{k}\right)^{\prime}$ be distributed according to a multinomial distribution $\operatorname{Mult}_{k}(n, \boldsymbol{\pi})$, where $\sum_{j=1}^{k} X_{j}=n, \sum_{j=1}^{k} \pi_{j}=1,0<\pi_{j}<1,(j=1, \ldots, k)$ and $\boldsymbol{\pi}=\left(\pi_{1}, \ldots, \pi_{k}\right)^{\prime}$ is an unknown probability vector. In order to test the null hypothesis

$$
H_{0}: \boldsymbol{\pi}=\boldsymbol{p}
$$

for some completely specified probability vector $\boldsymbol{p}=\left(p_{1}, \ldots, p_{k}\right)^{\prime}$, Zografos et al. (1990) introduced the $\phi$-divergence family of statistics

$$
C_{\phi}=\frac{2 n}{\phi^{\prime \prime}(1)}\left\{D_{\phi}\left(\frac{\boldsymbol{X}}{n}, \boldsymbol{p}\right)-\phi(1)\right\}, \quad\left(\phi^{\prime \prime}(1) \neq 0\right) .
$$

We consider a twice continuously differentiable convex function $\phi(t)$ for $t>0$ satisfying $\phi(1)=\phi^{\prime}(1)=0$ and $\phi^{\prime \prime}(1)=1$ (Pardo et al. (1999)) without loss of generality. The $\phi$-divergence family of statistics is thus defined by

$$
C_{\phi}=2 n D_{\phi}\left(\frac{\boldsymbol{X}}{n}, \boldsymbol{p}\right) \text {. }
$$

When we choose the following convex function $\phi_{a}^{P}$ as $\phi$ :

$$
\phi_{a}^{P}(t)= \begin{cases}\{a(a+1)\}^{-1}\left\{t^{a+1}-t+a(1-t)\right\} & (a \neq 0,-1) \\ t \ln t+1-t & (a=0) \\ -\ln t-1+t & (a=-1),\end{cases}
$$

the $\phi$-divergence statistics $C_{\phi}$ become the power divergence statistics $C_{\phi_{a}^{P}}$ (Cressie and Read (1984) and Read and Cressie (1988)). It is well known that the family of statistics $C_{\phi_{a}^{P}}$ includes Pearson's $X^{2}$ statistic $(a=1)$, the log-likelihood ratio statistic $(a=0)$, the Freeman-Tukey statistic $(a=-1 / 2)$, the modified loglikelihood ratio statistic $(a=-1)$, the Neyman modified $X^{2}$ statistic $(a=-2)$, and the statistic recommended by Cressie and Read $(a=2 / 3)$. Rukhin (1994) considered the family of statistics given by the following convex function $\phi_{a}^{R}$ as $\phi:$

$$
\phi_{a}^{R}(t)=\frac{(t-1)^{2}}{2\{a+t(1-a)\}}, \quad a \in[0,1] .
$$

Then the statistic based on $\phi_{a}^{R}$ is defined as

$$
C_{\phi_{a}^{R}}=n \sum_{j=1}^{k} \frac{\left(\hat{p}_{j}-p_{j}\right)^{2}}{a p_{j}+(1-a) \hat{p}_{j}}, \quad a \in[0,1],
$$

where $\hat{p}_{j}=X_{j} / n,(j=1, \ldots, k)$. The family of the statistics $C_{\phi_{a}^{R}}$ includes Pearson's $X^{2}$ statistic $(a=1)$ and the Neyman modified $X^{2}$ statistic $(a=$ $0)$. Furthermore, Lin (1991) considered the family of divergences given by the following convex function $\phi_{a}^{L}$ as $\phi$.

$$
\phi_{a}^{L}(t)=\{a(1-a)\}^{-1}\{a t \ln t-(a t+1-a) \ln (a t+1-a)\}, \quad a \in[0,1] .
$$


Then the statistic based on $\phi_{a}^{L}$ is defined as

$$
\begin{aligned}
C_{\phi_{a}^{L}}=\frac{2 n}{a(1-a)}\left\{-\sum_{j=1}^{k} s_{j} \ln s_{j}+(1-a) \sum_{j=1}^{k} p_{j} \ln p_{j}\right. & \\
& \left.+a \sum_{j=1}^{k} \hat{p}_{j} \ln \hat{p}_{j}\right\}, \quad a \in[0,1],
\end{aligned}
$$

where $s_{j}=a \hat{p}_{j}+(1-a) p_{j},(j=1, \ldots, k)$. The family of the statistics $C_{\phi_{a}^{L}}$ includes the log-likelihood ratio statistic $(a=0)$ and the modified log-likelihood ratio statistic $(a=1)$ by considering continuity in $a$. Under the null hypothesis $H_{0}$, statistics $C_{\phi}$ are asymptotically distributed according to a central chi-square distribution with $k-1$ degrees of freedom. Yarnold (1972) obtained an approximation based on asymptotic expansion for the null distribution of Pearson's $X^{2}$ statistic. In a similar fashion to $X^{2}$, asymptotic expansions for the null distributions of the log-likelihood ratio statistic and the Freeman-Tukey statistic were obtained by Siotani and Fujikoshi (1984), that of the power divergence statistic was obtained by Read (1984), and that of $C_{\phi}$ was obtained by Menéndez et al. (1997).

Against $H_{0}$, consider the local alternative hypothesis

$$
H_{1, n}: \pi_{j}=p_{j}+\frac{c_{j}}{\sqrt{n}} \quad \text { for all } \quad j=1, \ldots, k,
$$

where $\sum_{j=1}^{k} c_{j}=0$. It is known that $C_{\phi}$ are asymptotically distributed according to a noncentral chi-square distribution with $k-1$ degrees of freedom and noncentrality parameter

$$
\delta=\sum_{j=1}^{k} \frac{c_{j}^{2}}{p_{j}}
$$

under $H_{1, n}$. Taneichi et al. (2001) derived the local Edgeworth expansion under $H_{1, n}$ and obtained an expression of approximation for the distribution of $C_{\phi}$ under $H_{1, n}$. That expression consists of a term of multivariate Edgeworth expansion for a continuous distribution and a discontinuous term to account for the discontinuity in $\boldsymbol{X}$. The discontinuous term is expressed in a very complicated form, and it is too complicated to use in practice. Thus, using only the term of the multivariate Edgeworth expansion for a continuous distribution, Taneichi et al. (2001) proposed an approximation to the power of $C_{\phi}$. Based on numerical investigation, it was concluded that omission of the discontinuous term does not lead to a serious error in approximating the power of the test based on $C_{\phi}$.

On the other hand, consider a nonlocal (fixed) alternative hypothesis

$$
H_{1}: \boldsymbol{\pi}=\boldsymbol{q},
$$

where $\boldsymbol{q}=\left(q_{1}, \ldots, q_{k}\right)^{\prime}$ is a completely specified probability vector satisfying $0<q_{j}<1,(j=1, \ldots, k), \sum_{j=1}^{k} q_{j}=1, \boldsymbol{q} \neq \boldsymbol{p}$ and $\boldsymbol{q}$ does not depend 
on $n$. It is known that each $C_{\phi}$ is asymptotically distributed according to a normal distribution under $H_{1}$. We summarize here the results of studies on the limiting distribution of $C_{\phi}$ under $H_{1}$. Broffitt and Randles (1977) showed that an appropriate normalized Pearson's $X^{2}$ statistic is distributed according to the standard normal distribution for fixed $k$ and nonlocal alternatives. Read and Cressie (1988) extended the asymptotic normality to the power divergence statistics $C_{\phi_{a}^{P}}$. For the $\phi$-divergence statistic $C_{\phi}$, it was shown that

$$
\frac{C_{\phi}-2 n D_{\phi}(\boldsymbol{q}, \boldsymbol{p})}{2 \sqrt{n \sigma_{\phi}^{2}}} \stackrel{L}{\longrightarrow} N(0,1) \quad \text { as } \quad n \rightarrow \infty
$$

where

$$
\begin{aligned}
\sigma_{\phi}^{2} & =\sum_{j=1}^{k} q_{j}\left(\phi^{\prime}\left(r_{j}\right)\right)^{2}-\left(\sum_{j=1}^{k} q_{j} \phi^{\prime}\left(r_{j}\right)\right)^{2}, \\
r_{j} & =\frac{q_{j}}{p_{j}}, \quad(j=1, \ldots, k),
\end{aligned}
$$

and $\stackrel{L}{\longrightarrow}$ denotes convergence in distribution. Sekiya and Taneichi (2004) described a local Edgeworth expansion under $H_{1}$ and derived a multivariate Edgeworth expansion assuming a continuous distribution for the distribution of power divergence statistics $C_{\phi_{a}^{P}}$.

Recently, for power divergence statistics $C_{\phi_{a}^{P}}$, Ulyanov and Zubov (2009) evaluated the order of a discrete term of the distribution of the statistics under the simple null hypothesis $H_{0}$. By using the evaluation, they obtained an evaluation for the error of approximating the distribution of statistics to a chisquare distribution under the simple null hypothesis $H_{0}$. Furthermore, Assylbekov (2010) refined the evaluation. However, the method used to obtain their results is difficult to apply to the case of a distribution of statistics under alternative hypotheses $H_{1, n}$ or $H_{1}$ and for a more general multinomial model such as a contingency table.

In this paper, for practical consideration, we derive the expansion assuming a continuous distribution for the general $\phi$-divergence statistic $C_{\phi}$. In Section 2, we describe a local Edgeworth expansion under $H_{1}$ and derive the multivariate Edgeworth expansion assuming a continuous distribution for the distribution of $C_{\phi}$ under $H_{1}$. In Section 3, we apply the expansion to the power approximation for $C_{\phi}$ against $H_{1}$. Performance of the power approximation is numerically compared with that of other power approximations in the case of statistic $C_{\phi_{a}^{R}}$ given by (1.2) and statistic $C_{\phi_{a}^{L}}$ given by (1.3). 
2. Improvement of the limiting normal approximation for the distribution of $C_{\phi}$ under $H_{1}$

In this section, we consider the distribution of $C_{\phi}$ under $H_{1}$. Let $\boldsymbol{X}=$ $\left(X_{1}, \ldots, X_{k}\right)^{\prime}$ be distributed according to $\operatorname{Mult}_{k}(n, \boldsymbol{q})$. Let $r=k-1$,

$$
\begin{aligned}
Y_{j} & =\frac{X_{j}-n q_{j}}{\sqrt{n}}, \quad(j=1, \ldots, r), \\
\boldsymbol{Y} & =\left(Y_{1}, \ldots, Y_{r}\right)^{\prime}, \\
\tilde{\boldsymbol{q}} & =\left(q_{1}, \ldots, q_{r}\right)^{\prime},
\end{aligned}
$$

and

$$
\Lambda=\operatorname{diag}\left(q_{1}, \ldots, q_{r}\right)-\tilde{\boldsymbol{q}} \tilde{\boldsymbol{q}}^{\prime}
$$

Then

$$
E(\boldsymbol{Y})=\boldsymbol{O}, \quad \operatorname{Cov}(\boldsymbol{Y})=\Lambda,
$$

and the sample space of $\boldsymbol{Y}$ is the set

$$
L=\left\{\boldsymbol{y}=\left(y_{1}, \ldots, y_{r}\right)^{\prime}: \boldsymbol{y}=\frac{\boldsymbol{m}-n \tilde{\boldsymbol{q}}}{\sqrt{n}}, \boldsymbol{m} \in M\right\},
$$

where

$$
\begin{aligned}
M=\left\{\boldsymbol{m}=\left(m_{1}, \ldots, m_{r}\right)^{\prime}:\right. & \\
& \left.m_{j}(j=1, \ldots, r) \text { is a nonnegative integer, } \sum_{j=1}^{r} m_{j} \leq n\right\} .
\end{aligned}
$$

The local Edgeworth expansion of $\boldsymbol{Y}$ is given by the following theorem, which follows from Theorem 22.1 in Bhattacharya and Ranga Rao (1976) (see also Lemma 2.1 in Siotani and Fujikoshi (1984)).

TheOREM 1. For each $\boldsymbol{m} \in M$, let $\boldsymbol{y}=(\boldsymbol{m}-n \tilde{\boldsymbol{q}}) / \sqrt{n}$. Then

$$
\operatorname{Pr}\left\{\boldsymbol{Y}=\boldsymbol{y} \mid H_{1}\right\}=n^{-r / 2} g(\boldsymbol{y})\left\{1+\frac{1}{\sqrt{n}} h_{1}(\boldsymbol{y})+\frac{1}{n} h_{2}(\boldsymbol{y})+O\left(n^{-3 / 2}\right)\right\},
$$

where

$$
\begin{aligned}
& g(\boldsymbol{y})=(2 \pi)^{-r / 2}|\Lambda|^{-1 / 2} \exp \left(-\frac{1}{2} \boldsymbol{y}^{\prime} \Lambda^{-1} \boldsymbol{y}\right), \\
& h_{1}(\boldsymbol{y})=-\frac{1}{2} \sum_{j=1}^{k} \frac{y_{j}}{q_{j}}+\frac{1}{6} \sum_{j=1}^{k} \frac{y_{j}^{3}}{q_{j}^{2}}
\end{aligned}
$$




$$
h_{2}(\boldsymbol{y})=\frac{1}{2}\left\{h_{1}(\boldsymbol{y})\right\}^{2}+\frac{1}{12}-\frac{1}{12} \sum_{j=1}^{k} \frac{1}{q_{j}}+\frac{1}{4} \sum_{j=1}^{k} \frac{y_{j}^{2}}{q_{j}^{2}}-\frac{1}{12} \sum_{j=1}^{k} \frac{y_{j}^{4}}{q_{j}^{3}},
$$

and $y_{k}=-\sum_{j=1}^{r} y_{j}$.

By (2.1), the $\phi$-divergence statistics $C_{\phi}$ can be written as

$$
C_{\phi}(\boldsymbol{Y})=2 n \sum_{j=1}^{k} p_{j} \phi\left(\frac{\sqrt{n} Y_{j}+n q_{j}}{n p_{j}}\right),
$$

where $Y_{k}=-\sum_{j=1}^{r} Y_{j}$. Let

$$
S_{\phi}(\boldsymbol{Y})=\frac{C_{\phi}(\boldsymbol{Y})-2 n D_{\phi}(\boldsymbol{q}, \boldsymbol{p})}{2 \sqrt{n}} .
$$

From the asymptotic normality of $C_{\phi}$ under $H_{1}$, we know that the limiting distribution of $S_{\phi}(\boldsymbol{Y})$ is $N\left(0, \sigma_{\phi}^{2}\right)$. We consider the random variable $\tilde{\boldsymbol{Y}}$ which has the continuous probability density function

$$
g(\boldsymbol{y})\left\{1+\frac{1}{\sqrt{n}} h_{1}(\boldsymbol{y})+\frac{1}{n} h_{2}(\boldsymbol{y})\right\} .
$$

We also consider the distribution of $S_{\phi}(\tilde{\boldsymbol{Y}})$, which is given by substituting $\tilde{\boldsymbol{Y}}$ for $\boldsymbol{Y}$ in $S_{\phi}(\boldsymbol{Y})$. Let $F(x)$ be the cumulative distribution function of $S_{\phi}(\tilde{\boldsymbol{Y}})$. The multivariate Edgeworth expansion for $F(x)$ is given as follows.

Theorem 2. Let

$$
\begin{aligned}
F(x) & =\operatorname{Pr}\left\{S_{\phi}(\tilde{\boldsymbol{Y}}) \leq x \mid H_{1}\right\} \\
& =\int \cdots \int_{S_{\phi}(\boldsymbol{y}) \leq x} g(\boldsymbol{y})\left\{1+\frac{1}{\sqrt{n}} h_{1}(\boldsymbol{y})+\frac{1}{n} h_{2}(\boldsymbol{y})\right\} d y_{1} \cdots d y_{r} .
\end{aligned}
$$

If we assume that $\phi(t)$ is third time differentiable and $\phi^{\prime \prime \prime}(t)$ is continuous, then

$$
\begin{aligned}
F(x)= & \Phi(z)-\frac{1}{\sqrt{n \sigma_{\phi}^{2}}} \varphi(z)\left\{G_{1}^{\phi}+\frac{G_{2}^{\phi}}{\sigma_{\phi}^{2}}\left(z^{2}-1\right)\right\} \\
& -\frac{z}{n \sigma_{\phi}^{2}} \varphi(z)\left\{G_{3}^{\phi}+\frac{G_{4}^{\phi}}{\sigma_{\phi}^{2}}\left(z^{2}-3\right)+\frac{G_{5}^{\phi}}{\sigma_{\phi}^{4}}\left(z^{4}-10 z^{2}+15\right)\right\} \\
& +O\left(n^{-3 / 2}\right)
\end{aligned}
$$

where $\Phi(\cdot)$ and $\varphi(\cdot)$ denote the standard normal distribution function and its first derivative, respectively,

$$
G_{1}^{\phi}=\frac{1}{2}\left\{\sum_{j=1}^{k} \phi^{\prime \prime}\left(r_{j}\right) r_{j}-\sum_{j=1}^{k} \phi^{\prime \prime}\left(r_{j}\right) q_{j} r_{j}\right\}
$$




$$
\begin{aligned}
G_{2}^{\phi}= & \frac{1}{3}\left(\sum_{j=1}^{k} \phi^{\prime}\left(r_{j}\right) q_{j}\right)^{3}+\frac{1}{2}\left(\sum_{j=1}^{k} \phi^{\prime}\left(r_{j}\right) q_{j}\right)^{2}\left(\sum_{j=1}^{k} \phi^{\prime \prime}\left(r_{j}\right) q_{j} r_{j}\right) \\
& -\left(\sum_{j=1}^{k} \phi^{\prime}\left(r_{j}\right) q_{j}\right)\left\{\frac{1}{2} \sum_{j=1}^{k}\left(\phi^{\prime}\left(r_{j}\right)\right)^{2} q_{j}+\sum_{j=1}^{k} \phi^{\prime \prime}\left(r_{j}\right) \phi^{\prime}\left(r_{j}\right) q_{j} r_{j}\right\} \\
& +\frac{1}{6} \sum_{j=1}^{k}\left(\phi^{\prime}\left(r_{j}\right)\right)^{3} q_{j}+\frac{1}{2} \sum_{j=1}^{k} \phi^{\prime \prime}\left(r_{j}\right)\left(\phi^{\prime}\left(r_{j}\right)\right)^{2} q_{j} r_{j}, \\
G_{3}^{\phi}= & \frac{1}{2}\left(G_{1}^{\phi}\right)^{2}+\frac{1}{4}\left(\sum_{j=1}^{k} \phi^{\prime \prime}\left(r_{j}\right) q_{j} r_{j}\right)^{2}
\end{aligned}
$$$$
+\left(\sum_{j=1}^{k} \phi^{\prime}\left(r_{j}\right) q_{j}\right)\left\{-\frac{1}{2} \sum_{j=1}^{k} \phi^{\prime \prime}\left(r_{j}\right) r_{j}+\sum_{j=1}^{k} \phi^{\prime \prime}\left(r_{j}\right) q_{j} r_{j}\right.
$$$$
\left.-\frac{1}{2} \sum_{j=1}^{k} \phi^{\prime \prime \prime}\left(r_{j}\right) r_{j}^{2}+\frac{1}{2} \sum_{j=1}^{k} \phi^{\prime \prime \prime}\left(r_{j}\right) q_{j} r_{j}^{2}\right\}
$$$$
+\frac{1}{2} \sum_{j=1}^{k} \phi^{\prime \prime}\left(r_{j}\right) \phi^{\prime}\left(r_{j}\right) r_{j}-\sum_{j=1}^{k} \phi^{\prime \prime}\left(r_{j}\right) \phi^{\prime}\left(r_{j}\right) q_{j} r_{j}
$$$$
+\frac{1}{4} \sum_{j=1}^{k}\left(\phi^{\prime \prime}\left(r_{j}\right)\right)^{2} r_{j}{ }^{2}-\frac{1}{2} \sum_{j=1}^{k}\left(\phi^{\prime \prime}\left(r_{j}\right)\right)^{2} q_{j} r_{j}^{2}
$$

$$
\begin{aligned}
&+\frac{1}{2} \sum_{j=1}^{k} \phi^{\prime \prime \prime}\left(r_{j}\right) \phi^{\prime}\left(r_{j}\right) r_{j}{ }^{2}-\frac{1}{2} \sum_{j=1}^{k} \phi^{\prime \prime \prime}\left(r_{j}\right) \phi^{\prime}\left(r_{j}\right) q_{j} r_{j}^{2}, \\
& G_{4}^{\phi}=G_{1}^{\phi} G_{2}^{\phi}-\frac{1}{4}\left(\sum_{j=1}^{k} \phi^{\prime}\left(r_{j}\right) q_{j}\right)^{4}
\end{aligned}
$$

$$
\begin{aligned}
-\left(\sum_{j=1}^{k} \phi^{\prime}\left(r_{j}\right) q_{j}\right)^{3} & \left\{\sum_{j=1}^{k} \phi^{\prime \prime}\left(r_{j}\right) q_{j} r_{j}+\frac{1}{6} \sum_{j=1}^{k} \phi^{\prime \prime \prime}\left(r_{j}\right) q_{j} r_{j}{ }^{2}\right\} \\
+\left(\sum_{j=1}^{k} \phi^{\prime}\left(r_{j}\right) q_{j}\right)^{2}\{ & -\frac{1}{2}\left(\sum_{j=1}^{k} \phi^{\prime \prime}\left(r_{j}\right) q_{j} r_{j}\right)^{2}+\frac{1}{2} \sum_{j=1}^{k}\left(\phi^{\prime}\left(r_{j}\right)\right)^{2} q_{j} \\
& +2 \sum_{j=1}^{k} \phi^{\prime \prime}\left(r_{j}\right) \phi^{\prime}\left(r_{j}\right) q_{j} r_{j} \\
& \left.+\frac{1}{2} \sum_{j=1}^{k}\left(\phi^{\prime \prime}\left(r_{j}\right)\right)^{2} q_{j} r_{j}{ }^{2}+\frac{1}{2} \sum_{j=1}^{k} \phi^{\prime \prime \prime}\left(r_{j}\right) \phi^{\prime}\left(r_{j}\right) q_{j} r_{j}{ }^{2}\right\}
\end{aligned}
$$




$$
\begin{aligned}
+\left(\sum_{j=1}^{k} \phi^{\prime}\left(r_{j}\right) q_{j}\right)\{ & \left(\sum_{j=1}^{k} \phi^{\prime \prime}\left(r_{j}\right) q_{j} r_{j}\right) \\
& \times\left(\frac{1}{2} \sum_{j=1}^{k}\left(\phi^{\prime}\left(r_{j}\right)\right)^{2} q_{j}+\sum_{j=1}^{k} \phi^{\prime \prime}\left(r_{j}\right) \phi^{\prime}\left(r_{j}\right) q_{j} r_{j}\right) \\
& -\frac{1}{6} \sum_{j=1}^{k}\left(\phi^{\prime}\left(r_{j}\right)\right)^{3} q_{j}-\frac{3}{2} \sum_{j=1}^{k} \phi^{\prime \prime}\left(r_{j}\right)\left(\phi^{\prime}\left(r_{j}\right)\right)^{2} q_{j} r_{j} \\
& -\sum_{j=1}^{k}\left(\phi^{\prime \prime}\left(r_{j}\right)\right)^{2} \phi^{\prime}\left(r_{j}\right) q_{j} r_{j}^{2} \\
& \left.-\frac{1}{2} \sum_{j=1}^{k} \phi^{\prime \prime \prime}\left(r_{j}\right)\left(\phi^{\prime}\left(r_{j}\right)\right)^{2} q_{j} r_{j}{ }^{2}\right\} \\
- & \frac{1}{8}\left\{\sum_{j=1}^{k}\left(\phi^{\prime}\left(r_{j}\right)\right)^{2} q_{j}+2 \sum_{j=1}^{k} \phi^{\prime \prime}\left(r_{j}\right) \phi^{\prime}\left(r_{j}\right) q_{j} r_{j}\right\}^{2} \\
+ & \frac{1}{6} \sum_{j=1}^{k} \phi^{\prime \prime \prime}\left(r_{j}\right)\left(\phi^{\prime}\left(r_{j}\right)\right)^{3} q_{j} r_{j}^{2} \\
+ & \frac{1}{24} \sum_{j=1}^{k}\left(\phi^{\prime}\left(r_{j}\right)\right)^{4} q_{j}+\frac{1}{2} \sum_{j=1}^{k} \phi^{\prime \prime}\left(r_{j}\right)\left(\phi^{\prime}\left(r_{j}\right)\right)^{3} q_{j} r_{j} \\
+ & \frac{1}{2} \sum_{j=1}^{k}\left(\phi^{\prime \prime}\left(r_{j}\right)\right)^{2}\left(\phi^{\prime}\left(r_{j}\right)\right)^{2} q_{j} r_{j}{ }^{2},
\end{aligned}
$$

and

$$
G_{5}^{\phi}=\frac{1}{2}\left(G_{2}^{\phi}\right)^{2}
$$

with $\sigma_{\phi}^{2}$ and $r_{j}$ being defined by (1.7) and (1.8), respectively, and $z=x / \sigma_{\phi}$.

Proof. The characteristic function of $S_{\phi}(\tilde{\boldsymbol{Y}})$ is

$$
c(t)=\int_{-\infty}^{\infty} \cdots \int_{-\infty}^{\infty} \exp \left\{i t S_{\phi}(\boldsymbol{y})\right\} g(\boldsymbol{y})\left\{1+\frac{1}{\sqrt{n}} h_{1}(\boldsymbol{y})+\frac{1}{n} h_{2}(\boldsymbol{y})\right\} d y_{1} \cdots d y_{r} .
$$

We can expand $S_{\phi}(\boldsymbol{y})$ as

$$
S_{\phi}(\boldsymbol{y})=\sum_{j=1}^{r} \Theta_{j}^{\phi} y_{j}+\frac{1}{2 \sqrt{n}} \sum_{j=1}^{k} \phi^{\prime \prime}\left(r_{j}\right) \frac{y_{j}^{2}}{p_{j}}+\frac{1}{6 n} \sum_{j=1}^{k} \phi^{\prime \prime \prime}\left(r_{j}\right) \frac{y_{j}{ }^{3}}{p_{j}{ }^{2}}+O\left(n^{-3 / 2}\right),
$$

where

$$
\Theta_{j}^{\phi}=\phi^{\prime}\left(r_{j}\right)-\phi^{\prime}\left(r_{k}\right), \quad(j=1, \ldots, r) .
$$


Therefore, we have

$$
\begin{aligned}
c(t)= & \exp \left(-\frac{t^{2}}{2}\left(\boldsymbol{\Theta}^{\phi}\right)^{\prime} \Lambda \boldsymbol{\Theta}^{\phi}\right) \\
& \times \int_{-\infty}^{\infty} \cdots \int_{-\infty}^{\infty} g^{*}(\boldsymbol{y}) \gamma(\boldsymbol{y}) d y_{1} \cdots d y_{r}+O\left(n^{-3 / 2}\right),
\end{aligned}
$$

where

$$
\begin{aligned}
g^{*}(\boldsymbol{y})= & (2 \pi)^{-r / 2}|\Lambda|^{-1 / 2} \exp \left\{-\frac{1}{2}\left(\boldsymbol{y}-i t \Lambda \boldsymbol{\Theta}^{\phi}\right)^{\prime} \Lambda^{-1}\left(\boldsymbol{y}-i t \Lambda \boldsymbol{\Theta}^{\phi}\right)\right\} \\
\gamma(\boldsymbol{y})=1+ & \frac{1}{\sqrt{n}}\left\{\frac{i t}{2} \sum_{j=1}^{k} \phi^{\prime \prime}\left(r_{j}\right) \frac{y_{j}^{2}}{p_{j}}+h_{1}(\boldsymbol{y})\right\} \\
+ & \frac{1}{n}\left\{\frac{i t}{6} \sum_{j=1}^{k} \phi^{\prime \prime \prime}\left(r_{j}\right) \frac{y_{j}^{3}}{p_{j}{ }^{2}}+\frac{\left(i t^{2}\right)}{8}\left(\sum_{j=1}^{k} \phi^{\prime \prime}\left(r_{j}\right) \frac{y_{j}^{2}}{p_{j}}\right)^{2}\right. \\
& \left.+\frac{i t}{2} h_{1}(\boldsymbol{y}) \sum_{j=1}^{k} \phi^{\prime \prime}\left(r_{j}\right) \frac{y_{j}^{2}}{p_{j}}+h_{2}(\boldsymbol{y})\right\}
\end{aligned}
$$

and $\Theta^{\phi}=\left(\Theta_{1}^{\phi}, \ldots, \Theta_{r}^{\phi}\right)^{\prime}$. Carrying out the integral (2.3) with the aid of the moment formulae for a multivariate normal variate, we have

$$
\begin{aligned}
c(t)=\exp \left(-\frac{1}{2} t^{2} \sigma_{\phi}^{2}\right)[ & 1+\frac{1}{\sqrt{n}}\left\{(i t) G_{1}^{\phi}+(i t)^{3} G_{2}^{\phi}\right\} \\
& \left.+\frac{1}{n}\left\{(i t)^{2} G_{3}^{\phi}+(i t)^{4} G_{4}^{\phi}+(i t)^{6} G_{5}^{\phi}\right\}\right]+O\left(n^{-3 / 2}\right) .
\end{aligned}
$$

Inverting the equation above, we obtain the probability density function $f(x)$ of $S_{\phi}(\tilde{\boldsymbol{Y}})$ as

$$
\begin{aligned}
f(x)= & \frac{1}{\sqrt{\sigma_{\phi}^{2}}} \varphi(z)+\frac{1}{\sqrt{n}} \varphi(z)\left\{\frac{G_{1}^{\phi}}{\sigma_{\phi}^{2}} H_{1}(z)+\frac{G_{2}^{\phi}}{\sigma_{\phi}^{4}} H_{3}(z)\right\} \\
& +\frac{1}{n \sqrt{\sigma_{\phi}^{2}}} \varphi(z)\left\{\frac{G_{3}^{\phi}}{\sigma_{\phi}^{2}} H_{2}(z)+\frac{G_{4}^{\phi}}{\sigma_{\phi}^{4}} H_{4}(z)+\frac{G_{5}^{\phi}}{\sigma_{\phi}^{6}} H_{6}(z)\right\}+O\left(n^{-3 / 2}\right),
\end{aligned}
$$

where $H_{j}(\cdot)$ is the $j$ th Hermitian polynomial

$$
H_{j}(z)=(-1)^{j} \frac{1}{\varphi(z)}\left(\frac{d^{j}}{d z^{j}} \varphi(z)\right) .
$$

Consequently, the cumulative distribution function $F(x)$ of $S_{\phi}(\tilde{\boldsymbol{Y}})$ is obtained as (2.2). We have completed the proof of Theorem 2 .

If we use only the leading term of the right-hand side of (2.2), we have

$$
F(x)=\Phi(z)+O\left(n^{-1 / 2}\right) .
$$




\section{Application and numerical investigation}

By considering the distribution of $S_{\phi}(\tilde{\boldsymbol{Y}})$ as the distribution of $S_{\phi}(\boldsymbol{Y})$, we derive the following two approximations of power $\operatorname{Pr}\left\{C_{\phi}(\boldsymbol{Y})>c_{0}^{\phi} \mid H_{1}\right\}$ of $C_{\phi}(\boldsymbol{Y})$ against $H_{1}$ by using (2.2). The first approximation is given by

$$
1-\Phi(v)+\frac{1}{\sqrt{n \sigma_{\phi}^{2}}} \varphi(v)\left\{G_{1}^{\phi}+\frac{G_{2}^{\phi}}{\sigma_{\phi}^{2}}\left(v^{2}-1\right)\right\},
$$

where

$$
v=\frac{c_{0}^{\phi}-2 n D_{\phi}(\boldsymbol{q}, \boldsymbol{p})}{2 \sqrt{n \sigma_{\phi}^{2}}}
$$

and $c_{0}^{\phi}$ is the critical value for $C_{\phi}$. This approximation is obtained by considering (2.2) up to order of $n^{-1 / 2}$. We refer to this power approximation method as the $A N(m)$ approximation. The second approximation is given by

$$
\begin{aligned}
1-\Phi(v) & +\frac{1}{\sqrt{n \sigma_{\phi}^{2}}} \varphi(v)\left\{G_{1}^{\phi}+\frac{G_{2}^{\phi}}{\sigma_{\phi}^{2}}\left(v^{2}-1\right)\right\} \\
& +\frac{v}{n \sigma_{\phi}^{2}} \varphi(v)\left\{G_{3}^{\phi}+\frac{G_{4}^{\phi}}{\sigma_{\phi}^{2}}\left(v^{2}-3\right)+\frac{G_{5}^{\phi}}{\sigma_{\phi}^{4}}\left(v^{4}-10 v^{2}+15\right)\right\} .
\end{aligned}
$$

This approximation is obtained by considering (2.2) up to order of $n^{-1}$. We refer to this power approximation method as the $A N(h)$ approximation.

We compare the performance of the $A N(m)$ and $A N(h)$ approximations with that of other power approximations. Drost et al. (1989) proposed two power approximations for the power divergence statistic $C_{\phi_{a}^{P}}, A^{a}$ and $B^{a}$. The $A^{a}$ approximation is constructed from a linear combination of mutually independent noncentral chi-square random variables, and the $B^{a}$ approximation is constructed from a linear expression of a single noncentral chi-square random variable that has the same first moment as $A^{a}$. Taneichi et al. (2001) derived $A^{\phi}$ and $B^{\phi}$ approximations on the basis of $A^{a}$ and $B^{a}$ approximations for the $\phi$-divergence family of statistics $C_{\phi}$.

Let

$$
\begin{aligned}
& A_{j}=\phi^{\prime}\left(r_{j}\right), \quad(j=1, \ldots, k), \\
& B_{j}=\frac{1}{p_{j}} \phi^{\prime \prime}\left(r_{j}\right), \quad(j=1, \ldots, k), \\
& \boldsymbol{q}^{1 / 2}=\left(q_{1}^{1 / 2}, \ldots, q_{k}^{1 / 2}\right)^{\prime}, \\
& Q=\operatorname{diag}\left(\left(B_{1} q_{1}\right)^{1 / 2}, \ldots,\left(B_{k} q_{k}\right)^{1 / 2}\right),
\end{aligned}
$$

$\gamma_{1}, \ldots, \gamma_{r}$ are non-zero eigenvalues and $S$ is the $k \times k$ orthogonal matrix of eigenvectors of $Q\left(E-\boldsymbol{q}^{1 / 2}\left(\boldsymbol{q}^{1 / 2}\right)^{\prime}\right) Q$ :

$$
S^{\prime} Q\left(E-\boldsymbol{q}^{1 / 2}\left(\boldsymbol{q}^{1 / 2}\right)^{\prime}\right) Q S=\operatorname{diag}\left(\gamma_{1}, \ldots, \gamma_{r}, 0\right),
$$


where $E$ denotes the $k$-dimensional unit matrix.

Then the $A^{\phi}$ approximation to the power of $C_{\phi}$ against the alternative hypothesis $H_{1}$ given by (1.5) is represented as

$$
1-\operatorname{Pr}\left\{\sum_{j=1}^{k-1} \gamma_{j} \chi_{1}^{2}\left(\frac{\left(\xi_{j}\right)^{2}}{\gamma_{j}}\right)<c_{0}^{\phi}-2 n \nu+\sum_{j=1}^{k-1}\left(\xi_{j}\right)^{2}\right\},
$$

where

$$
\begin{aligned}
& \left(\xi_{1}, \ldots, \xi_{k}\right)^{\prime}=S^{\prime} Q \boldsymbol{\mu}, \\
& \boldsymbol{\mu}=n^{1 / 2}\left(\frac{A_{1}}{B_{1} \sqrt{q_{1}}}, \ldots, \frac{A_{k}}{B_{k} \sqrt{q_{k}}}\right)^{\prime}, \\
& \nu=D_{\phi}(\boldsymbol{q}, \boldsymbol{p}),
\end{aligned}
$$

where $\chi_{1}^{2}(\delta)$ is a chi-square random variable with 1 degree of freedom and noncentrality parameter $\delta$, and $D_{\phi}(\cdot, \cdot)$ is given by $(1.1)$.

The $B^{\phi}$ approximation to the power of $C_{\phi}$ against the alternative hypothesis $H_{1}$ given by (1.5) is represented as

$$
1-\operatorname{Pr}\left\{\chi_{r}^{2}(\omega)<\frac{c_{0}^{\phi}-2 n \nu+\bar{B} \omega}{\bar{B}}\right\},
$$

where

$$
\bar{B}=\frac{1}{k-1} \sum_{j=1}^{k} B_{j} q_{j}\left(1-q_{j}\right)
$$

and

$$
\omega=n \bar{B}^{-2}\left\{\sum_{j=1}^{k} A_{j}^{2} q_{j}-\left(\sum_{j=1}^{k} A_{j} q_{j}\right)^{2}\right\} .
$$

Sekiya et al. (1999) proposed NT approximation, which is a normal approximation based on the normalizing transformation of a power divergence statistic. Taneichi et al. (2001) derived an $N^{\phi}$ approximation based on the method of the NT approximation for the power divergence statistic for the $\phi$-divergence family of statistics $C_{\phi}$. The $N^{\phi}$ approximation to the power of $C_{\phi}$ against the alternative hypothesis $H_{1}$ given by (1.5) is represented as

$$
1-\Phi\left(\sqrt{\frac{n}{\nu_{2}}}\left\{\psi\left(\frac{c_{0}^{\phi}}{2 n}\right)-\frac{1}{n}\left(\nu_{1}-\frac{\nu_{3}}{6 \nu_{2}}\right)\right\}\right),
$$

where

$$
\psi(x)= \begin{cases}\frac{\nu}{\zeta+1}\left(\left(\frac{x}{\nu}\right)^{\zeta+1}-1\right) & (\zeta \neq-1) \\ \nu \ln \frac{x}{\nu} & (\zeta=-1)\end{cases}
$$


Table 1. Values of $E r$ for the $R_{0.5}$ statistic when $k=4, \pi=p^{(1)}$ and alternative hypotheses are in $Q_{l}(l=1,2,3)$ and arbitrary.

\begin{tabular}{|c|c|c|c|c|c|c|c|}
\hline$n$ & $\chi^{2}$ & $A^{\phi}$ & $B^{\phi}$ & $N^{\phi}$ & $A E$ & $A N(m)$ & $A N(h)$ \\
\hline \multicolumn{8}{|c|}{$Q_{1}$} \\
\hline $5 k$ & 0.042267 & $\underline{0.008041}$ & 0.008728 & 0.102908 & 0.016269 & 0.064929 & 0.043094 \\
\hline $6 k$ & 0.083914 & 0.040939 & 0.039016 & 0.104563 & $\underline{0.027471}$ & 0.076088 & 0.036797 \\
\hline $8 k$ & 0.091101 & 0.027192 & 0.024616 & 0.080194 & $\underline{0.024519}$ & 0.056578 & 0.030508 \\
\hline $10 k$ & 0.079879 & 0.012802 & 0.010821 & 0.083912 & $\underline{0.010804}$ & 0.045919 & 0.025671 \\
\hline \multicolumn{8}{|c|}{$Q_{2}$} \\
\hline $5 k$ & 0.158130 & $\underline{0.019257}$ & 0.033956 & 0.033969 & 0.042920 & 0.052703 & 0.037563 \\
\hline $6 k$ & 0.184125 & 0.037407 & 0.038586 & 0.021644 & 0.060576 & 0.026920 & $\underline{0.019488}$ \\
\hline $8 k$ & 0.133935 & 0.010371 & 0.011988 & $\underline{0.009698}$ & 0.029118 & 0.009838 & 0.010215 \\
\hline $10 k$ & 0.087939 & 0.009338 & 0.010270 & 0.005938 & 0.009060 & 0.012111 & $\underline{0.002858}$ \\
\hline \multicolumn{8}{|c|}{$Q_{3}$} \\
\hline $5 k$ & 0.086395 & 0.015851 & 0.020009 & $\underline{0.009033}$ & 0.026678 & 0.021775 & 0.011980 \\
\hline $6 k$ & 0.076164 & 0.009338 & 0.010582 & 0.013161 & 0.027859 & 0.008709 & $\underline{0.008145}$ \\
\hline $8 k$ & 0.030895 & 0.007392 & 0.010024 & 0.003709 & 0.017181 & 0.012948 & $\underline{0.001153}$ \\
\hline $10 k$ & 0.013837 & 0.004755 & 0.008947 & 0.002229 & 0.016993 & 0.013377 & $\underline{0.000258}$ \\
\hline \multicolumn{8}{|c|}{ Arbitrary } \\
\hline $5 k$ & 0.102145 & $\underline{0.014416}$ & 0.023351 & 0.075076 & 0.028649 & 0.056071 & 0.037001 \\
\hline $6 k$ & 0.126202 & 0.036814 & 0.036425 & 0.083851 & 0.041674 & 0.056762 & $\underline{0.028994}$ \\
\hline $8 k$ & 0.102344 & 0.021184 & $\underline{0.019793}$ & 0.065830 & 0.025039 & 0.043270 & 0.023788 \\
\hline $10 k$ & 0.079303 & 0.011516 & 0.010654 & 0.070156 & 0.010263 & 0.037348 & 0.019960 \\
\hline
\end{tabular}

According to the numerical values in Tables $1-5$, we have the following results for the $R_{0.5}$ statistic.

1. The $A N(h)$ approximation is good for $Q_{3}$, which is a set of alternatives distant from the null hypothesis.

2. The $A N(h)$ approximation is good for $p^{(2)}, p^{(3)}, p^{(4)}$ and $p^{(5)}$ in an arbitrary case.

3. The $A N(h)$ approximation performs better than the $A N(m)$ approximation.

According to Tables $6-10$, we have the following results for the $L_{0.1}$ statistic.

1. The $A N(h)$ approximation is better than the other six approximations for $Q_{3}$.

2. The $A N(h)$ approximation performs better than other approximations for $p^{(2)}, p^{(3)}$ and $p^{(5)}$ in an arbitrary case.

3. The $A N(h)$ approximation performs better than the $A N(m)$ approximation.

$$
\begin{aligned}
& \zeta=-\frac{\nu \nu_{3}}{3\left(\nu_{2}\right)^{2}}, \\
& \nu_{1}=\frac{1}{2} \sum_{j=1}^{k} B_{j} M_{j j}^{(2)}, \\
& \nu_{2}=\sum_{j=1}^{k} \sum_{l=1}^{k} A_{j} A_{l} M_{j l}^{(2)}, \\
& \nu_{3}=\sum_{j=1}^{k} \sum_{l=1}^{k} \sum_{m=1}^{k}\left(A_{j} A_{l} A_{m} M_{j l m}^{(3)}+3 A_{j} A_{l} B_{m} M_{j m}^{(2)} M_{l m}^{(2)}\right), \\
& M_{j l}^{(2)}=\delta_{j l} q_{j}-q_{j} q_{l},
\end{aligned}
$$


Table 2. Values of $E r$ for the $R_{0.5}$ statistic when $k=4, \pi=p^{(2)}$ and alternative hypotheses are in $Q_{l}(l=1,2,3)$ and arbitrary.

\begin{tabular}{rccccccc}
\hline$n$ & $\chi^{2}$ & $A^{\phi}$ & $B^{\phi}$ & $N^{\phi}$ & $A E$ & $A N(m)$ & $A N(h)$ \\
\hline $5 k$ & 0.080773 & 0.051033 & 0.051318 & 0.051034 & $\underline{0.037789}$ & 0.088908 & 0.065634 \\
$6 k$ & 0.058833 & $\underline{0.010078}$ & 0.020124 & 0.053461 & 0.018312 & 0.052311 & 0.028333 \\
$8 k$ & 0.080078 & 0.023952 & 0.023210 & 0.043796 & 0.022870 & 0.046254 & $\underline{0.019546}$ \\
$10 k$ & 0.081118 & 0.019932 & 0.019092 & 0.037204 & 0.019786 & 0.036316 & $\underline{0.017424}$ \\
& & & & $Q_{2}$ & & & \\
$5 k$ & 0.142714 & 0.058404 & 0.059582 & 0.035187 & 0.068475 & 0.036568 & $\underline{0.028485}$ \\
$6 k$ & 0.104642 & 0.011780 & 0.025846 & $\underline{0.011537}$ & 0.030836 & 0.023342 & 0.016560 \\
$8 k$ & 0.087366 & 0.013979 & 0.014404 & 0.012550 & 0.022144 & 0.010755 & $\underline{0.008544}$ \\
$10 k$ & 0.062798 & 0.007885 & 0.007509 & 0.009393 & 0.013466 & 0.007468 & $\underline{0.004594}$ \\
& & & & $Q_{3}$ & & & \\
$5 k$ & 0.096097 & 0.013989 & 0.015151 & 0.013016 & 0.039734 & 0.011002 & $\underline{0.009252}$ \\
$6 k$ & 0.056412 & 0.010579 & 0.011162 & $\underline{0.004012}$ & 0.025147 & 0.013589 & 0.006687 \\
$8 k$ & 0.030006 & 0.005964 & 0.006718 & 0.006950 & 0.026769 & 0.008966 & $\underline{0.003498}$ \\
$10 k$ & 0.022518 & 0.003655 & 0.007001 & 0.003642 & 0.024328 & 0.010603 & $\underline{0.001088}$ \\
\hline & & & \multicolumn{7}{c}{ Arbitrary } & & & \\
$5 k$ & 0.111639 & 0.048204 & 0.049067 & 0.033441 & 0.052186 & 0.057493 & $\underline{0.028521}$ \\
$6 k$ & 0.079680 & $\underline{0.010740}$ & 0.018410 & 0.030521 & 0.025845 & 0.036102 & 0.020310 \\
$8 k$ & 0.081544 & 0.018905 & 0.018597 & 0.024694 & 0.024081 & 0.031730 & $\underline{0.013769}$ \\
$10 k$ & 0.073006 & 0.015513 & 0.014854 & 0.032629 & 0.017986 & 0.026174 & $\underline{0.012384}$ \\
\hline
\end{tabular}

For $R_{a}(a=0.0 \sim 1.0)$ statistics, we have the following results.

1. The $A N(h)$ and $N^{\phi}$ approximations are better than other approximations for $a=0.0,0.1,0.2$ in an arbitrary case.

2. For $a=0.3,0.4, A N(h)$ and $B^{\phi}$ approximations are better than other approximations in an arbitrary case.

3. In the seven power approximations, $A N(h)$ and $A^{\phi}$ approximations are better than other approximations for $a=0.6,0.7$ in an arbitrary case.

4. For $a=0.8,0.9,1.0$, the $A^{\phi}$ approximation is better than the other six approximations in an arbitrary case.

5. The $A N(h)$ approximation is a good approximation, especially for $a=0.4,0.5,0.6,0.7,0.8,0.9$ in the $Q_{3}$ case.

6. The $A N(h)$ approximation performs better than the $A N(m)$ approximation.

and

$$
M_{j l m}^{(3)}=\delta_{j l} \delta_{j m} q_{j}-\left(\delta_{j l} q_{j} q_{m}+\delta_{j m} q_{j} q_{l}+\delta_{l m} q_{j} q_{l}\right)+2 q_{j} q_{l} q_{m} .
$$

By (3.1), (3.2), (3.3), (3.4) and (3.5), we obtained the power approximation against $H_{1}$.

On the other hand, Taneichi et al. (2002) proposed the $A E$ approximation, which is based on multivariate Edgeworth expansion assuming a continuous distribution for the distribution of $C_{\phi_{a}^{P}}$ under $H_{1, n}$ given by (1.4). Taneichi et al. (2001) derived the $A E$ approximation for the distribution of a $\phi$-divergence statistic. The expression of the $A E$ approximation for the power of $C_{\phi}$ against 
Table 3. Values of $E r$ for the $R_{0.5}$ statistic when $k=4, \pi=p^{(3)}$ and alternative hypotheses are in $Q_{l}(l=1,2,3)$ and arbitrary.

\begin{tabular}{|c|c|c|c|c|c|c|c|}
\hline$n$ & $\chi^{2}$ & $A^{\phi}$ & $B^{\phi}$ & $N^{\phi}$ & $A E$ & $A N(m)$ & $A N(h)$ \\
\hline \multicolumn{8}{|c|}{$Q_{1}$} \\
\hline $5 k$ & 0.048615 & 0.029430 & $\underline{0.026263}$ & 0.083458 & 0.044519 & 0.062808 & 0.035057 \\
\hline $6 k$ & 0.049613 & 0.023083 & $\underline{0.020387}$ & 0.062194 & 0.033961 & 0.055609 & 0.032460 \\
\hline $8 k$ & 0.078913 & 0.022438 & $\underline{0.021182}$ & 0.054360 & 0.022162 & 0.053890 & 0.027135 \\
\hline $10 k$ & 0.107247 & 0.034363 & 0.034932 & 0.060677 & 0.039536 & 0.053451 & $\underline{0.026454}$ \\
\hline \multicolumn{8}{|c|}{$Q_{2}$} \\
\hline $5 k$ & 0.152525 & 0.032091 & 0.032347 & $\underline{0.014656}$ & 0.065019 & 0.018980 & 0.015949 \\
\hline $6 k$ & 0.136704 & 0.022260 & 0.025188 & 0.013632 & 0.035946 & 0.020456 & $\underline{0.013070}$ \\
\hline $8 k$ & 0.134928 & 0.011430 & 0.014668 & 0.011254 & 0.028610 & $\underline{0.008107}$ & 0.008340 \\
\hline $10 k$ & 0.118579 & 0.009481 & 0.007639 & 0.005586 & 0.029159 & 0.011006 & $\underline{0.005320}$ \\
\hline \multicolumn{8}{|c|}{$Q_{3}$} \\
\hline $5 k$ & 0.118788 & 0.009312 & 0.013883 & 0.006203 & 0.104387 & 0.011812 & $\underline{0.005688}$ \\
\hline $6 k$ & 0.098506 & 0.010161 & 0.013299 & 0.011132 & 0.091359 & 0.009141 & $\underline{0.008646}$ \\
\hline $8 k$ & 0.080790 & 0.004135 & 0.006478 & 0.006406 & 0.076357 & 0.007961 & $\underline{0.003801}$ \\
\hline $10 k$ & 0.067067 & 0.004301 & 0.009396 & $\underline{0.001680}$ & 0.053925 & 0.012614 & 0.002245 \\
\hline \multicolumn{8}{|c|}{ Arbitrary } \\
\hline $5 k$ & 0.118868 & 0.024126 & 0.024248 & 0.045743 & 0.079670 & 0.033202 & $\underline{0.020579}$ \\
\hline $6 k$ & 0.101999 & $\underline{0.018509}$ & 0.019515 & 0.037989 & 0.060586 & 0.032759 & 0.019828 \\
\hline $8 k$ & 0.103064 & $\underline{0.015742}$ & 0.016298 & 0.043464 & 0.041232 & 0.034565 & 0.018362 \\
\hline $10 k$ & 0.104862 & 0.024746 & 0.025154 & 0.043005 & 0.036153 & 0.038284 & $\underline{0.018876}$ \\
\hline
\end{tabular}

For $L_{a}(a=0.0 \sim 1.0)$ statistics, we have the following results.

1. The $A N(h)$ and $A^{\phi}$ approximations are better than the other five approximations for $a=0.0,0.1$, 0.2 and 0.3 in an arbitrary case.

2. For $a=0.4,0.5,0.6$ and 0.7 , the $A N(h)$ approximation is a good approximation in an arbitrary case.

3. The $A N(h)$ and $N^{\phi}$ approximations perform better than the other five approximations for $a=0.8$ and 0.9 in an arbitrary case.

4. The $A N(h)$ approximation is a good approximation, especially for $a=0.0,0.1,0.2,0.3$ and 0.4 in the $Q_{3}$ case.

5. The $A N(h)$ approximation performs better than the $A N(m)$ approximation.

$H_{1, n}$ is represented as

$$
\begin{gathered}
1-\operatorname{Pr}\left\{\chi_{r}^{2}(\delta)<c_{0}^{\phi}\right\}-\frac{1}{6 \sqrt{n}} \sum_{j=0}^{3} w_{j} \operatorname{Pr}\left\{\chi_{r+2 j}^{2}(\delta)<c_{0}^{\phi}\right\} \\
-\frac{1}{72 n} \sum_{j=0}^{6} v_{j} \operatorname{Pr}\left\{\chi_{r+2 j}^{2}(\delta)<c_{0}^{\phi}\right\},
\end{gathered}
$$

where

$$
\begin{aligned}
& w_{0}=2 S_{2}^{3}, \\
& w_{1}=-3\left\{\left(S_{1}^{1}+S_{2}^{3}\right)+T^{3} S_{1}^{1}\right\},
\end{aligned}
$$


Table 4. Values of $E r$ for the $R_{0.5}$ statistic when $k=4, \pi=p^{(4)}$ and alternative hypotheses are in $Q_{l}(l=1,2,3)$ and arbitrary.

\begin{tabular}{rccccccc}
\hline$n$ & $\chi^{2}$ & $A^{\phi}$ & $B^{\phi}$ & $N^{\phi}$ & $A E$ & $A N(m)$ & $A N(h)$ \\
\hline $5 k$ & 0.038166 & 0.027559 & $\underline{0.026861}$ & 0.061536 & 0.041470 & 0.071714 & 0.035245 \\
$6 k$ & 0.064714 & 0.027334 & $\underline{0.026560}$ & 0.052533 & 0.030146 & 0.067045 & 0.034706 \\
$8 k$ & 0.106397 & 0.041042 & 0.042707 & 0.040404 & 0.042263 & 0.061131 & $\underline{0.029081}$ \\
$10 k$ & 0.106827 & 0.013065 & $\underline{0.012078}$ & 0.055586 & 0.028429 & 0.037834 & 0.024681 \\
& & & \multicolumn{5}{c}{$Q_{2}$} \\
$5 k$ & 0.111375 & 0.032688 & 0.035081 & $\underline{0.019449}$ & 0.042811 & 0.029333 & 0.021235 \\
$6 k$ & 0.118166 & 0.025078 & 0.026249 & 0.018130 & 0.037964 & 0.017923 & $\underline{0.016549}$ \\
$8 k$ & 0.118273 & 0.013974 & 0.015600 & 0.012979 & 0.026790 & 0.011034 & $\underline{0.008888}$ \\
$10 k$ & 0.101244 & 0.007702 & $\underline{0.006403}$ & 0.006722 & 0.022649 & 0.010561 & 0.007652 \\
& & & \multicolumn{7}{c}{$Q_{3}$} & & & \\
$5 k$ & 0.098476 & 0.016156 & 0.018490 & 0.016172 & 0.074393 & $\underline{0.010734}$ & 0.013211 \\
$6 k$ & 0.089007 & 0.008875 & 0.010221 & 0.011517 & 0.066224 & 0.007769 & $\underline{0.007746}$ \\
$8 k$ & 0.070454 & 0.005430 & 0.006216 & 0.007491 & 0.056739 & 0.007701 & $\underline{0.004002}$ \\
$10 k$ & 0.051043 & 0.004895 & 0.009297 & $\underline{0.002945}$ & 0.035825 & 0.012418 & 0.003794 \\
\hline & & & \multicolumn{7}{c}{ Arbitrary } & & & \\
$5 k$ & 0.094951 & 0.024126 & 0.025508 & 0.031530 & 0.059836 & 0.033816 & $\underline{0.020239}$ \\
$6 k$ & 0.095339 & 0.018969 & 0.019553 & 0.027492 & 0.054523 & 0.028944 & $\underline{0.017488}$ \\
$8 k$ & 0.099198 & 0.022437 & 0.023374 & 0.023038 & 0.041761 & 0.032261 & $\underline{0.016064}$ \\
$10 k$ & 0.091979 & 0.009375 & $\underline{0.009054}$ & 0.038540 & 0.027072 & 0.023412 & 0.015543 \\
\hline
\end{tabular}

$$
\begin{aligned}
w_{2}= & 3 S_{1}^{1}+T^{3}\left(3 S_{1}^{1}-S_{2}^{3}\right) \\
w_{3}= & \left(1+T^{3}\right) S_{2}^{3} \\
v_{0}= & 6\left(1-S_{1}^{0}\right)-18 S_{3}^{4}+4\left(S_{2}^{3}\right)^{2} \\
v_{1}= & \left(-k^{2}-2 k+3 S_{1}^{0}\right)+18 T^{3}\left(-k^{2}+S_{1}^{0}\right)+9 T^{4}\left(2 k-1-S_{1}^{0}\right) \\
& +3\left(T^{3}\right)^{2}\left(-3 k^{2}-6 k+4+5 S_{1}^{0}\right)+3\left\{-6 k S_{1}^{2}+6 S_{2}^{2}+11 S_{3}^{4}\right. \\
& \left.-3\left(S_{1}^{2}\right)^{2}-4\left(S_{2}^{3}\right)^{2}-4 S_{1}^{1} S_{2}^{3}\right\}+6 T^{3}\left\{-3 k S_{1}^{2}+3 S_{2}^{2}-2 S_{1}^{1} S_{2}^{3}\right\} \\
v_{2}= & 8\left(k^{2}+2 k-1-2 S_{1}^{0}\right)+12 T^{3}\left(3 k^{2}+3 k-2-4 S_{1}^{0}\right)+9 T^{4}\left(-2 k+1+S_{1}^{0}\right) \\
& +6\left(T^{3}\right)^{2}\left(3 k^{2}+6 k-4-5 S_{1}^{0}\right)+3\left\{6 k S_{1}^{2}+3\left(S_{1}^{1}\right)^{2}+3\left(S_{2}^{3}\right)^{2}+10 S_{1}^{1} S_{2}^{3}\right\} \\
& +2 T^{3}\left\{9 S_{3}^{4}+9\left(S_{1}^{1}\right)^{2}-9\left(S_{1}^{2}\right)^{2}-2\left(S_{2}^{3}\right)^{2}+15 S_{1}^{1} S_{2}^{3}\right\}+18 T^{4}\left\{S_{1}^{2}-S_{2}^{2}\right\} \\
& +9\left(T^{3}\right)^{2}\left\{-2(k+2) S_{1}^{2}+4 S_{2}^{2}+\left(S_{1}^{1}\right)^{2}\right\} \\
v_{3}= & 3\left(1+T^{3}\right)^{2}\left(-3 k^{2}-6 k+4+5 S_{1}^{0}\right)+2\left\{9(k+2) S_{1}^{2}-27 S_{2}^{2}-9 S_{3}^{4}\right. \\
& \left.-9\left(S_{1}^{1}\right)^{2}+9\left(S_{1}^{2}\right)^{2}+2\left(S_{2}^{3}\right)^{2}-9 S_{1}^{1} S_{2}^{3}\right\}+2 T^{3}\left\{9(3 k+4) S_{1}^{2}-45 S_{2}^{2}-9 S_{3}^{4}\right. \\
& \left.-18\left(S_{1}^{1}\right)^{2}+9\left(S_{1}^{2}\right)^{2}+5\left(S_{2}^{3}\right)^{2}-6 S_{1}^{1} S_{2}^{3}\right\}+3 T^{4}\left\{-6 S_{1}^{2}+6 S_{2}^{2}-S_{3}^{4}\right\} \\
& +3\left(T^{3}\right)^{2}\left\{12(k+2) S_{1}^{2}-24 S_{2}^{2}+3 S_{3}^{4}-6\left(S_{1}^{1}\right)^{2}-3\left(S_{1}^{2}\right)^{2}+2 S_{1}^{1} S_{2}^{3}\right\} \\
v_{4}= & 3\left\{-6(k+2) S_{1}^{2}+12 S_{2}^{2}-2 S_{3}^{4}+3\left(S_{1}^{1}\right)^{2}-2\left(S_{2}^{3}\right)^{2}-2 S_{1}^{1} S_{2}^{3}\right\} \\
& +6 T^{3}\left\{-6(k+2) S_{1}^{2}+12 S_{2}^{2}-3 S_{3}^{4}+3\left(S_{1}^{1}\right)^{2}+3\left(S_{1}^{2}\right)^{2}-\left(S_{2}^{3}\right)^{2}-3 S_{1}^{1} S_{2}^{3}\right\}
\end{aligned}
$$


Table 5. Values of $E r$ for the $R_{0.5}$ statistic when $k=4, \pi=p^{(5)}$ and alternative hypotheses are in $Q_{l}(l=1,2,3)$ and arbitrary.

\begin{tabular}{|c|c|c|c|c|c|c|c|}
\hline$n$ & $\chi^{2}$ & $A^{\phi}$ & $B^{\phi}$ & $N^{\phi}$ & $A E$ & $A N(m)$ & $A N(h)$ \\
\hline \multicolumn{8}{|c|}{$Q_{1}$} \\
\hline $5 k$ & 0.044482 & 0.024932 & $\underline{0.022576}$ & 0.070262 & 0.032252 & 0.061835 & 0.032815 \\
\hline $6 k$ & 0.067414 & 0.032518 & 0.030674 & 0.056282 & $\underline{0.028858}$ & 0.059027 & 0.030262 \\
\hline $8 k$ & 0.100328 & 0.040601 & 0.040705 & 0.045488 & 0.039802 & 0.059119 & $\underline{0.028959}$ \\
\hline $10 k$ & 0.086140 & 0.015541 & $\underline{0.014511}$ & 0.036950 & 0.018041 & 0.039054 & 0.018991 \\
\hline \multicolumn{8}{|c|}{$Q_{2}$} \\
\hline $5 k$ & 0.124811 & 0.029449 & 0.028948 & $\underline{0.019071}$ & 0.047091 & 0.025758 & 0.019959 \\
\hline $6 k$ & 0.126329 & 0.026115 & 0.026612 & 0.015109 & 0.039806 & 0.015802 & $\underline{0.014386}$ \\
\hline $8 k$ & 0.110150 & 0.011854 & 0.012767 & 0.010918 & 0.026571 & 0.008754 & $\underline{0.007362}$ \\
\hline $10 k$ & 0.083496 & 0.006406 & 0.006401 & 0.007542 & 0.018744 & 0.007963 & $\underline{0.004071}$ \\
\hline \multicolumn{8}{|c|}{$Q_{3}$} \\
\hline $5 k$ & 0.098434 & 0.009743 & 0.012487 & $\underline{0.009239}$ & 0.064349 & 0.013256 & 0.009443 \\
\hline $6 k$ & 0.082612 & 0.007421 & 0.009612 & 0.008095 & 0.060910 & 0.008729 & $\underline{0.004875}$ \\
\hline $8 k$ & 0.061500 & 0.005606 & 0.007686 & 0.004031 & 0.051555 & 0.010201 & $\underline{0.001715}$ \\
\hline $10 k$ & 0.044984 & 0.003303 & 0.007845 & 0.002759 & 0.039167 & 0.011103 & $\underline{0.001046}$ \\
\hline \multicolumn{8}{|c|}{ Arbitrary } \\
\hline $5 k$ & 0.095221 & 0.022473 & $\underline{0.021812}$ & 0.042556 & 0.049499 & 0.038507 & 0.021853 \\
\hline $6 k$ & 0.095942 & 0.025626 & 0.025145 & 0.034438 & 0.044181 & 0.036501 & $\underline{0.019354}$ \\
\hline $8 k$ & 0.097214 & 0.027151 & 0.027341 & 0.031606 & 0.037262 & 0.039334 & $\underline{0.019453}$ \\
\hline $10 k$ & 0.081747 & 0.011908 & $\underline{0.011362}$ & 0.026651 & 0.020498 & 0.028663 & 0.013898 \\
\hline
\end{tabular}

$$
\begin{aligned}
& +3 T^{4} S_{3}^{4}+\left(T^{3}\right)^{2}\left\{-18(k+2) S_{1}^{2}+36 S_{2}^{2}-18 S_{3}^{4}+9\left(S_{1}^{1}\right)^{2}\right. \\
& \left.+18\left(S_{1}^{2}\right)^{2}+\left(S_{2}^{3}\right)^{2}-12 S_{1}^{1} S_{2}^{3}\right\}, \\
v_{5}= & 3\left\{3 S_{3}^{4}-3\left(S_{1}^{2}\right)^{2}+2 S_{1}^{1} S_{2}^{3}\right\}+2 T^{3}\left\{9 S_{3}^{4}-9\left(S_{1}^{2}\right)^{2}-\left(S_{2}^{3}\right)^{2}+6 S_{1}^{1} S_{2}^{3}\right\} \\
& +\left(T^{3}\right)^{2}\left\{9 S_{3}^{4}-9\left(S_{1}^{2}\right)^{2}-2\left(S_{2}^{3}\right)^{2}+6 S_{1}^{1} S_{2}^{3}\right\}, \\
v_{6}= & \left(1+T^{3}\right)^{2}\left(S_{2}^{3}\right)^{2}, \\
S_{m}^{l}= & \sum_{j=1}^{k}\left(c_{j}\right)^{l} /\left(p_{j}\right)^{m},
\end{aligned}
$$

and $T^{l}=\phi^{(l)}(1)$. If we use only the first two terms of (3.6), then we have

$$
1-\operatorname{Pr}\left\{\chi_{r}^{2}(\delta)<c_{0}^{\phi}\right\}
$$

which is a noncentral chi-square approximation. When we put $c_{j}=\sqrt{n}\left(q_{j}-p_{j}\right)$, $(j=1, \ldots, k)$, expressions (3.6) and (3.7) represent the $A E$ and noncentral $\chi^{2}$ approximations to the power of $C_{\phi}$ against $H_{1}$ given by (1.5).

We consider $C_{\phi_{a}^{R}}$ given by (1.2) and $C_{\phi_{a}^{L}}$ given by (1.3) as concrete $\phi$ divergence statistics. Hereafter, we call them $R_{a}$ statistics and $L_{a}$ statistics, respectively. If we put $\phi^{\prime}(x)=2^{-1}(x-1)(a+x(1-a)+1)(a+x(1-a))^{-2}$, $\phi^{\prime \prime}(x)=(a+x(1-a))^{-3}$ and $\phi^{\prime \prime \prime}(x)=-3(1-a)(a+x(1-a))^{-4}$ in $(3.1)$ and 
Table 6. Values of $E r$ for the $L_{0.1}$ statistic when $k=4, \pi=p^{(1)}$ and alternative hypotheses are in $Q_{l}(l=1,2,3)$ and arbitrary.

\begin{tabular}{|c|c|c|c|c|c|c|c|}
\hline$n$ & $\chi^{2}$ & $A^{\phi}$ & $B^{\phi}$ & $N^{\phi}$ & $A E$ & $A N(m)$ & $A N(h)$ \\
\hline \multicolumn{8}{|c|}{$Q_{1}$} \\
\hline $5 k$ & 0.036495 & $\underline{0.004295}$ & 0.005243 & 0.100749 & 0.012843 & 0.064217 & 0.047649 \\
\hline $6 k$ & 0.080365 & 0.041302 & 0.040255 & 0.097540 & $\underline{0.036550}$ & 0.078985 & 0.037054 \\
\hline $8 k$ & 0.086773 & 0.029153 & $\underline{0.028244}$ & 0.090201 & 0.031834 & 0.059757 & 0.030650 \\
\hline $10 k$ & 0.075125 & 0.012070 & $\underline{0.010902}$ & 0.085371 & 0.016466 & 0.045351 & 0.025972 \\
\hline \multicolumn{8}{|c|}{$Q_{2}$} \\
\hline $5 k$ & 0.148746 & $\underline{0.029657}$ & 0.072152 & 0.036234 & 0.051725 & 0.059631 & 0.044640 \\
\hline $6 k$ & 0.063679 & 0.177310 & 0.035668 & $\underline{0.020435}$ & 0.072654 & 0.029027 & 0.021475 \\
\hline $8 k$ & 0.130002 & 0.014000 & 0.025253 & $\underline{0.010989}$ & 0.037138 & 0.014941 & 0.012599 \\
\hline $10 k$ & 0.085631 & 0.013124 & 0.020861 & $\underline{0.003547}$ & 0.012969 & 0.016971 & 0.005073 \\
\hline \multicolumn{8}{|c|}{$Q_{3}$} \\
\hline $5 k$ & 0.080064 & 0.024691 & 0.044568 & 0.009813 & 0.023640 & 0.032719 & $\underline{0.007970}$ \\
\hline $6 k$ & 0.071363 & 0.011411 & 0.018118 & $\underline{0.009854}$ & 0.024337 & 0.014648 & 0.010511 \\
\hline $8 k$ & 0.028245 & 0.008573 & 0.008775 & 0.003131 & 0.013605 & 0.013569 & $\underline{0.002953}$ \\
\hline $10 k$ & 0.013377 & 0.006404 & 0.007271 & $\underline{0.000821}$ & 0.011682 & 0.012823 & 0.001885 \\
\hline \multicolumn{8}{|c|}{ Arbitrary } \\
\hline $5 k$ & 0.095318 & $\underline{0.020708}$ & 0.102225 & 0.071197 & 0.032211 & 0.058040 & 0.041337 \\
\hline $6 k$ & 0.103939 & $\underline{0.025182}$ & 0.065664 & 0.069101 & 0.033111 & 0.053641 & 0.026445 \\
\hline $8 k$ & 0.090088 & $\underline{0.017449}$ & 0.047478 & 0.072134 & 0.022837 & 0.043131 & 0.021865 \\
\hline $10 k$ & 0.076466 & $\underline{0.012836}$ & 0.025895 & 0.070792 & 0.015491 & 0.037543 & 0.020208 \\
\hline
\end{tabular}

(3.2), we can obtain the coefficients $\sigma_{\phi}^{2}, G_{1}^{\phi}, \ldots, G_{5}^{\phi}$ of $A N(m)$ and $A N(h)$ approximations for $R_{a}$ statistics. If we put

$$
\begin{aligned}
\phi^{\prime}(x) & = \begin{cases}(1-a)^{-1} \ln (x /(a x+1-a)) & (a \neq 1,0) \\
\ln x & (a=0) \\
-x^{-1}+1 & (a=1),\end{cases} \\
\phi^{\prime \prime}(x) & = \begin{cases}(1-a)^{-1}\left(x^{-1}-a(a x+1-a)^{-1}\right) & (a \neq 1,0) \\
x^{-1} & (a=0) \\
x^{-2} & (a=1),\end{cases}
\end{aligned}
$$

and

$$
\phi^{\prime \prime \prime}(x)= \begin{cases}(1-a)^{-1}\left(-x^{-2}+a^{2}(a x+1-a)^{-2}\right) & (a \neq 1,0) \\ -x^{-2} & (a=0) \\ -2 x^{-3} & (a=1)\end{cases}
$$

in (3.1) and (3.2), we can obtain the coefficients of $A N(m)$ and $A N(h)$ approximations for $L_{a}$ statistics. Similarly, if we put $T^{3}=3(a-1)$ and $T^{4}=12(a-1)^{2}$ in (3.6), we obtain the coefficients of the $A E$ approximation for the $R_{a}$ statistic, and if we put $T^{3}=-(a+1)$ and $T^{4}=2\left(a^{2}+a+1\right)$ in (3.6), we obtain the 
Table 7. Values of $E r$ for the $L_{0.1}$ statistic when $k=4, \pi=p^{(2)}$ and alternative hypotheses are in $Q_{l}(l=1,2,3)$ and arbitrary.

\begin{tabular}{|c|c|c|c|c|c|c|c|}
\hline$n$ & $\chi^{2}$ & $A^{\phi}$ & $B^{\phi}$ & $N^{\phi}$ & $A E$ & $A N(m)$ & $A N(h)$ \\
\hline \multicolumn{8}{|c|}{$Q_{1}$} \\
\hline $5 k$ & 0.092420 & 0.064645 & 0.063841 & 0.065054 & 0.063427 & 0.099946 & $\underline{0.053759}$ \\
\hline $6 k$ & 0.054942 & $\underline{0.011614}$ & 0.026262 & 0.061178 & 0.018912 & 0.053618 & 0.030464 \\
\hline $8 k$ & 0.080382 & 0.026706 & 0.035874 & 0.049150 & 0.033238 & 0.047222 & $\underline{0.020727}$ \\
\hline $10 k$ & 0.070747 & $\underline{0.015968}$ & 0.034751 & 0.044895 & 0.019547 & 0.034900 & 0.016839 \\
\hline \multicolumn{8}{|c|}{$Q_{2}$} \\
\hline $5 k$ & 0.155924 & 0.066879 & 0.066192 & 0.044740 & 0.096565 & 0.048234 & $\underline{0.038333}$ \\
\hline $6 k$ & 0.101113 & 0.016570 & 0.039577 & $\underline{0.010770}$ & 0.036602 & 0.027041 & 0.014226 \\
\hline $8 k$ & 0.088277 & 0.012942 & 0.019476 & 0.011595 & 0.028619 & 0.012351 & $\underline{0.009637}$ \\
\hline $10 k$ & 0.056979 & 0.010098 & 0.012611 & 0.009230 & 0.012823 & 0.012049 & $\underline{0.005433}$ \\
\hline \multicolumn{8}{|c|}{$Q_{3}$} \\
\hline $5 k$ & 0.097303 & 0.016797 & 0.025714 & $\underline{0.012189}$ & 0.043469 & 0.017548 & 0.013469 \\
\hline $6 k$ & 0.053102 & 0.014168 & 0.022316 & 0.005414 & 0.019946 & 0.018227 & $\underline{0.005016}$ \\
\hline $8 k$ & 0.027371 & 0.007356 & 0.007040 & 0.004979 & 0.019090 & 0.011241 & $\underline{0.002952}$ \\
\hline $10 k$ & 0.016179 & 0.003878 & 0.004113 & 0.004032 & 0.018571 & 0.009233 & $\underline{0.001682}$ \\
\hline \multicolumn{8}{|c|}{ Arbitrary } \\
\hline $5 k$ & 0.119433 & 0.057972 & 0.064664 & 0.042642 & 0.072775 & 0.068856 & $\underline{0.037773}$ \\
\hline $6 k$ & 0.075613 & $\underline{0.014261}$ & 0.065762 & 0.030564 & 0.027683 & 0.038013 & 0.019896 \\
\hline $8 k$ & 0.081503 & 0.020691 & 0.041172 & 0.022929 & 0.031145 & 0.032772 & $\underline{0.014551}$ \\
\hline $10 k$ & 0.064233 & 0.013220 & 0.031754 & 0.036793 & 0.017311 & 0.025713 & $\underline{0.011932}$ \\
\hline
\end{tabular}

coefficient of the $A E$ approximation for the $L_{a}$ statistic. If we consider that $\phi_{a}^{R}$ and $\phi_{a}^{L}$ are $\phi$ in (3.3), (3.4), (3.5), we obtain $A^{\phi}, B^{\phi}$ and $N^{\phi}$ approximations for $R_{a}$ and $L_{a}$ statistics, respectively.

For $k=4$, we consider the equiprobable null hypothesis $H_{0}^{(1)}: \boldsymbol{\pi}=\boldsymbol{p}^{(1)}$, where $\boldsymbol{p}^{(1)}=\left(p_{1}^{(1)}, \ldots, p_{k}^{(1)}\right)^{\prime}=(1 / k, \ldots, 1 / k)^{\prime}$. We also consider four null hypotheses $H_{0}^{(l)}: \boldsymbol{\pi}=\boldsymbol{p}^{(l)},(l=2, \ldots, 5)$, where

$$
\begin{aligned}
& \boldsymbol{p}^{(2)}=(0.2,0.2,0.2,0.4)^{\prime}, \\
& \boldsymbol{p}^{(3)}=(0.1,0.3,0.3,0.3)^{\prime}, \\
& \boldsymbol{p}^{(4)}=(0.125,0.125,0.375,0.375)^{\prime} \quad \text { and } \\
& \boldsymbol{p}^{(5)}=(0.125,0.25,0.25,0.375)^{\prime} .
\end{aligned}
$$

For each null hypothesis, we randomly generate $M=1000$ alternative hypotheses $H_{1}^{(i)}: \boldsymbol{\pi}=\boldsymbol{q}^{(i)},(i=1, \ldots, M)$. Under each alternative $H_{1}^{(i)}$, we compute the true power $T P(i)$ of the randomized size- $\alpha$ test and approximate power $A P(i)$ of each approximation. For computing all approximate powers, we use the exact critical value obtained by direct enumeration. We investigate the performance of 
Table 8. Values of $E r$ for the $L_{0.1}$ statistic when $k=4, \pi=p^{(3)}$ and alternative hypotheses are in $Q_{l}(l=1,2,3)$ and arbitrary.

\begin{tabular}{|c|c|c|c|c|c|c|c|}
\hline$n$ & $\chi^{2}$ & $A^{\phi}$ & $B^{\phi}$ & $N^{\phi}$ & $A E$ & $A N(m)$ & $A N(h)$ \\
\hline \multicolumn{8}{|c|}{$Q_{1}$} \\
\hline $5 k$ & 0.064809 & 0.044752 & 0.043892 & 0.079204 & $\underline{0.031484}$ & 0.087999 & 0.046115 \\
\hline $6 k$ & 0.056915 & 0.025681 & 0.025611 & 0.080625 & $\underline{0.018200}$ & 0.068501 & 0.051985 \\
\hline $8 k$ & 0.076202 & 0.025348 & $\underline{0.024751}$ & 0.052311 & 0.034605 & 0.058779 & 0.030908 \\
\hline $10 k$ & 0.092600 & 0.032878 & 0.032418 & 0.062600 & 0.046879 & 0.055446 & $\underline{0.026312}$ \\
\hline \multicolumn{8}{|c|}{$Q_{2}$} \\
\hline $5 k$ & 0.151771 & 0.047297 & 0.117857 & $\underline{0.027494}$ & 0.071496 & 0.031972 & 0.027772 \\
\hline $6 k$ & 0.120834 & 0.025233 & 0.183083 & $\underline{0.019043}$ & 0.036847 & 0.034538 & 0.020476 \\
\hline $8 k$ & 0.117913 & 0.010850 & 0.054797 & 0.006803 & 0.040770 & 0.012103 & $\underline{0.005180}$ \\
\hline $10 k$ & 0.095191 & 0.010114 & 0.017228 & 0.008083 & 0.025443 & 0.012303 & $\underline{0.003911}$ \\
\hline \multicolumn{8}{|c|}{$Q_{3}$} \\
\hline $5 k$ & 0.080918 & 0.013205 & 0.105940 & 0.014572 & 0.087022 & 0.013885 & $\underline{0.013003}$ \\
\hline $6 k$ & 0.054208 & 0.010766 & 0.157480 & 0.003301 & 0.058391 & 0.015522 & $\underline{0.003289}$ \\
\hline $8 k$ & 0.062854 & 0.005000 & 0.005223 & 0.004278 & 0.052339 & 0.008700 & $\underline{0.000977}$ \\
\hline $10 k$ & 0.044398 & 0.003359 & 0.002654 & 0.005243 & 0.043260 & 0.006261 & $\underline{0.001681}$ \\
\hline \multicolumn{8}{|c|}{ Arbitrary } \\
\hline $5 k$ & 0.100720 & 0.033151 & 0.097989 & 0.047234 & 0.072159 & 0.042818 & $\underline{0.027829}$ \\
\hline $6 k$ & 0.081140 & $\underline{0.020773}$ & 0.143315 & 0.038110 & 0.045353 & 0.041733 & 0.024476 \\
\hline $8 k$ & 0.089227 & 0.018098 & 0.033112 & 0.035830 & 0.040342 & 0.037828 & $\underline{0.017849}$ \\
\hline $10 k$ & 0.085549 & 0.024301 & 0.024875 & 0.045170 & 0.037272 & 0.039524 & $\underline{0.018550}$ \\
\hline
\end{tabular}

each approximation based on the index

$$
E r=\sqrt{\frac{1}{M} \sum_{i=1}^{M}\{A P(i)-T P(i)\}^{2}} .
$$

We investigate $a=0.0 \sim 1.0$ by step 0.1 for each $L_{a}$ and $R_{a}$ statistic and consider the alternative hypotheses that are included in the set $Q_{l}(l=1,2,3)$ defined as follows:

$$
\begin{aligned}
& Q_{1}=\left\{\boldsymbol{q}=\left(q_{1}, \ldots, q_{k}\right)^{\prime}: \Delta(\boldsymbol{q}) \leq \frac{1}{k}\right\} \\
& Q_{2}=\left\{\boldsymbol{q}=\left(q_{1}, \ldots, q_{k}\right)^{\prime}: \frac{1}{k}<\Delta(\boldsymbol{q}) \leq \frac{2}{k}\right\}
\end{aligned}
$$

and

$$
Q_{3}=\left\{\boldsymbol{q}=\left(q_{1}, \ldots, q_{k}\right)^{\prime}: \frac{2}{k}<\Delta(\boldsymbol{q})\right\}
$$

where

$$
\Delta(\boldsymbol{q})=\sum_{j=1}^{k} \frac{\left(q_{j}-p_{j}^{(l)}\right)^{2}}{p_{j}^{(l)}}, \quad(l=1, \ldots, 5)
$$


Table 9. Values of $E r$ for the $L_{0.1}$ statistic when $k=4, \pi=p^{(4)}$ and alternative hypotheses are in $Q_{l}(l=1,2,3)$ and arbitrary.

\begin{tabular}{|c|c|c|c|c|c|c|c|}
\hline$n$ & $\chi^{2}$ & $A^{\phi}$ & $B^{\phi}$ & $N^{\phi}$ & $A E$ & $A N(m)$ & $A N(h)$ \\
\hline \multicolumn{8}{|c|}{$Q_{1}$} \\
\hline $5 k$ & 0.044910 & 0.033668 & 0.033916 & 0.065593 & $\underline{0.019006}$ & 0.084347 & 0.056746 \\
\hline $6 k$ & 0.077079 & 0.041991 & 0.041883 & 0.069761 & $\underline{0.037414}$ & 0.085138 & 0.056466 \\
\hline $8 k$ & 0.102990 & 0.049026 & 0.048529 & 0.071619 & 0.062548 & 0.068770 & $\underline{0.035864}$ \\
\hline $10 k$ & 0.079444 & $\underline{0.010706}$ & 0.028274 & 0.065943 & 0.026717 & 0.040293 & 0.024901 \\
\hline \multicolumn{8}{|c|}{$Q_{2}$} \\
\hline $5 k$ & 0.096514 & 0.036453 & 0.037146 & $\underline{0.024316}$ & 0.055979 & 0.032156 & 0.027831 \\
\hline $6 k$ & 0.110231 & 0.028991 & 0.046904 & $\underline{0.021684}$ & 0.056538 & 0.025424 & 0.022004 \\
\hline $8 k$ & 0.101343 & 0.011416 & 0.016756 & 0.011383 & 0.038766 & 0.009505 & $\underline{0.008304}$ \\
\hline $10 k$ & 0.072468 & 0.006561 & 0.006484 & 0.009948 & 0.019562 & 0.008905 & $\underline{0.005241}$ \\
\hline \multicolumn{8}{|c|}{$Q_{3}$} \\
\hline $5 k$ & 0.077116 & 0.015195 & 0.120588 & 0.016853 & 0.061929 & $\underline{0.014246}$ & 0.014676 \\
\hline $6 k$ & 0.068545 & 0.011220 & 0.080301 & 0.009735 & 0.055147 & 0.012884 & $\underline{0.007391}$ \\
\hline $8 k$ & 0.055114 & 0.004702 & 0.004626 & 0.004734 & 0.039554 & 0.007786 & $\underline{0.001334}$ \\
\hline $10 k$ & 0.031636 & 0.003713 & 0.003054 & 0.005405 & 0.030775 & 0.006835 & $\underline{0.002290}$ \\
\hline \multicolumn{8}{|c|}{ Arbitrary } \\
\hline $5 k$ & 0.077271 & $\underline{0.024336}$ & 0.073689 & 0.036740 & 0.056259 & 0.037356 & 0.026686 \\
\hline $6 k$ & 0.080683 & 0.025545 & 0.077422 & 0.030133 & 0.051903 & 0.041568 & $\underline{0.024879}$ \\
\hline $8 k$ & 0.084361 & 0.025541 & 0.025231 & 0.044349 & 0.044489 & 0.034821 & $\underline{0.019577}$ \\
\hline $10 k$ & 0.064293 & 0.007782 & $\underline{0.007576}$ & 0.043843 & 0.024291 & 0.023716 & 0.015663 \\
\hline
\end{tabular}

Note that the alternative hypotheses in set $Q_{1}$ are those for which the distance is very close to the null hypothesis, $Q_{2}$ is a set of alternative hypotheses with moderate distance from the null hypothesis and $Q_{3}$ is a set far from the null hypothesis. We also consider arbitrary alternative hypotheses. Some values of $E r$ for the level of significance $\alpha=0.05, k=4$, and sample size $n=5 k, 6 k$, $8 k$ and $10 k$ are listed in Tables $1-10$. Tables $1-5$ are for the $R_{a}$ statistic when $a=0.5$ and Tables $6-10$ are for the $L_{a}$ statistic when $a=0.1$. In each table, the underlined value indicates that the corresponding approximation is the best of all for each $n$.

\section{Concluding remarks}

In this paper, we derived a multivariate Edgeworth expansion assuming a continuous distribution for the distribution of $C_{\phi}$ under $H_{1}$ and applied the expansion to the power approximation for $C_{\phi}$ against $H_{1}$. According to the results of a numerical investigation presented in Section 3 , we conclude that the $A N(h)$ approximation performs better than the other approximaitons when $0.3 \leq a \leq 0.6$ for $R_{a}$ statistics and $0 \leq a \leq 0.9$ for $L_{a}$ statistics in an arbitrary case. The $A N(h)$ approximation is good for both $R_{a}$ and $L_{a}$ statistics in the $Q_{3}$ case, which means that the $A N(h)$ approximation is better than the other six approximations when the alternatives are far from the null hypothesis. 
Table 10. Values of $E r$ for the $L_{0.1}$ statistic when $k=4, \pi=p^{(5)}$ and alternative hypotheses are in $Q_{l}(l=1,2,3)$ and arbitrary.

\begin{tabular}{rccccccc}
\hline$n$ & $\chi^{2}$ & $A^{\phi}$ & $B^{\phi}$ & $N^{\phi}$ & $A E$ & $A N(m)$ & $A N(h)$ \\
\hline $5 k$ & 0.050371 & 0.031640 & 0.031230 & 0.070705 & $\underline{0.019022}$ & 0.075182 & 0.046495 \\
$6 k$ & 0.068190 & 0.034617 & 0.034106 & 0.049210 & 0.032760 & 0.068103 & $\underline{0.031619}$ \\
$8 k$ & 0.087374 & 0.034667 & 0.034137 & 0.047933 & 0.045436 & 0.056245 & $\underline{0.026740}$ \\
$10 k$ & 0.073920 & $\underline{0.013962}$ & 0.014152 & 0.039243 & 0.021301 & 0.039265 & 0.020760 \\
& & & & $Q_{2}$ & & & \\
$5 k$ & 0.120858 & 0.031825 & 0.111414 & $\underline{0.018582}$ & 0.052777 & 0.030813 & 0.022296 \\
$6 k$ & 0.115920 & 0.023399 & 0.084618 & 0.014429 & 0.052254 & 0.022255 & $\underline{0.013797}$ \\
$8 k$ & 0.094047 & 0.012667 & 0.028457 & 0.009155 & 0.030111 & 0.014416 & $\underline{0.006898}$ \\
$10 k$ & 0.069007 & 0.009167 & 0.014909 & 0.010236 & 0.016834 & 0.011461 & $\underline{0.005933}$ \\
& & & & $Q_{3}$ & & & \\
$5 k$ & 0.080353 & 0.014268 & 0.178507 & 0.011646 & 0.054853 & 0.016258 & $\underline{0.010168}$ \\
$6 k$ & 0.071263 & 0.010393 & 0.050573 & 0.006505 & 0.045879 & 0.013670 & $\underline{0.004814}$ \\
$8 k$ & 0.046669 & 0.006311 & 0.006230 & 0.005254 & 0.036892 & 0.009771 & $\underline{0.002624}$ \\
$10 k$ & 0.030239 & 0.004190 & 0.003525 & 0.006148 & 0.032289 & 0.007149 & $\underline{0.002980}$ \\
\hline & & & \multicolumn{7}{c}{ Arbitrary } & & & \\
$5 k$ & 0.088473 & 0.025725 & 0.117313 & 0.036187 & 0.046536 & 0.044176 & $\underline{0.024624}$ \\
$6 k$ & 0.087776 & 0.026180 & 0.081930 & 0.030372 & 0.043702 & 0.042844 & $\underline{0.019436}$ \\
$8 k$ & 0.081705 & 0.022923 & 0.036113 & 0.031512 & 0.037193 & 0.037951 & $\underline{0.017726}$ \\
$10 k$ & 0.067791 & $\underline{0.011270}$ & 0.018141 & 0.028748 & 0.020327 & 0.028611 & 0.015294 \\
\hline
\end{tabular}

We also found in the case of alternative hypotheses, the $A N(h)$ approximation, that is, the approximation based on expansion (2.2) up to order of $n^{-1}$, greatly improves the $A N(m)$ approximation, that is, the approximation based on expansion (2.2), up to an order of $n^{-1 / 2}$. In the future, we will search for a powerful statistic among $\phi$-divergence statistics based on the power approximation $A N(h)$.

\section{Acknowledgements}

The authors are very grateful to the reviewers for their valuable comments and suggestions.

\section{REFERENCES}

Ali, S. M. and Silvey, S. D. (1966). A general class of coefficients of divergence of one distribution from another, J. Roy. Statist. Soc. B, 28, 131-142.

Assylbekov, Z. (2010). Convergence rate of multinomial goodness-of-fit statistics to chi-square distribution, Hiroshima Math. J., 40, 115-131.

Bhattacharya, R. N. and Ranga Rao, R. (1976). Normal Approximation and Asymptotic Expansions, Wiley, New York.

Broffitt, J. D. and Randles, R. H. (1977). A power approximation for the chi-square goodnessof-fit test: simple hypothesis case, J. Amer. Statist. Assoc., 72, 604-607.

Cressie, N. and Read, T. R. C. (1984). Multinomial goodness-of-fit tests, J. R. Statist. Soc. B, 46, 440-464. 
Csiszár, I. (1967). Information-type measures of difference of probability distributions and indirect observations, Studia Sci. Math. Hungar., 2, 299-318.

Drost, F. C., Kallenberg, W. C. M., Moore, D. S. and Oosterhoff, J. (1989). Power approximations to multinomial tests of fit, J. Amer. Statist. Assoc., 84, 130-141.

Lin, J. (1991). Divergence measures based on the Shannon entropy, IEEE Trans. Information Theory, 37, 145-151.

Menéndez, M. L., Pardo, J. A., Pardo, L. and Pardo, M. C. (1997). Asymptotic approximations for the distributions of the $(h, \phi)$-divergence goodness-of-fit statistics: Application to Renyi's statistic, Kybernetes, 26(4), 442-452.

Pardo, L., Pardo, M. C. and Zografos, K. (1999). Homogeneity for multinomial populations based on $\phi$-divergences, J. Japan Statist. Soc., 29, 213-228.

Read, T. R. C. (1984). Closer asymptotic approximations for the distributions of the power divergence goodness-of-fit statistics, Ann. Inst. Statist. Math., 36, 59-69.

Read, T. R. C and Cressie, N. A. C. (1988), Goodness-of-fit Statistics for Discrete Multivariate Data, Springer-Verlag.

Rukhin, A. L. (1994). Optimal estimator for the mixture parameter by the method of moments and information affinity, in Trans. 12th Prague Conference on Information Theory, 214-219.

Sekiya, Y. and Taneichi, N. (2004). Improvement of approximations for the distributions of multinomial goodness-of-fit statistics under nonlocal alternatives, J. Multivar. Anal., 91, 199-223.

Sekiya, Y., Taneichi, N. and Imai, H. (1999). A power approximation for the multinomial goodness-of-fit test based on a normalizing transformation, J. Japan Statist. Soc., 29, 7987.

Siotani, M. and Fujikoshi, Y. (1984). Asymptotic approximations for the distributions of multinomial goodness-of-fit statistics, Hiroshima Math. J., 14, 115-124.

Taneichi, N., Sekiya, Y. and Suzukawa, A. (2001). An asymptotic approximation for the distribution of $\phi$-divergence multinomial goodness-of-fit statistic under local alternatives, $J$. Japan Statist. Soc., 31(2), 207-224.

Taneichi, N., Sekiya, Y. and Suzukawa, A. (2002). Asymptotic approximations for the distributions of the multinomial goodness-of-fit statistics under local alternatives, J. Multivar. Anal., 81(2), 335-359.

Ulyanov, V. V. and Zubov, V. N. (2009). Refinement on the convergence of one family of goodness-of-fit statistics to chi-squared distribution, Hiroshima Math. J., 39, 133-161.

Yarnold, J. K. (1972). Asymptotic approximations for the probability that a sum of lattice random vectors lies in a convex set, Ann. Math. Statist., 43, 1566-1580.

Zografos, K., Ferentions, K. and Papaioannou, T. (1990). $\phi$-divergence statistics: Sampling properties and multinomial goodness-of-fit and divergence tests, Commun. Statist.-Theory Meth., 19, 1785-1802. 